\title{
Revisiting unexploited antibiotics in search of new antibacterial drug candidates: the case of MSD-819 (6-chloro-2-quinoxalinecarboxylic acid 1,4-dioxide)
}

\author{
Nicola Ooi and Alex J O’Neill \\ The Journal of Antibiotics (2017) 70, 317-319; doi:10.1038/ja.2016.140; published online 30 November 2016
}

The emergence and spread of antibiotic resistance in bacterial pathogens is dramatically undermining our ability to treat infection, precipitating a public health crisis. ${ }^{1}$ The situation is particularly grave in the case of infections caused by the ESKAPE pathogens (Enterococcus faecium, Staphylococcus aureus, Klebsiella pneumoniae, Acinetobacter baumannii, Pseudomonas aeruginosa, and Enterobacter species), 2,3 a group of organisms whose name reflects their ability to 'escape' the action of existing antibacterial drugs. While the problem presented by these pathogens could be effectively addressed by developing novel antibacterial agents active against them, this is proving easier said than done; the field of antibacterial drug discovery is now 30 years into a 'Discovery Void', a period during which no new classes addressing the ESKAPE pathogens have successfully progressed from discovery to clinic. ${ }^{4}$

One approach to identifying new antibacterial drug candidates involves revisiting known natural product antibiotics that have not been exploited for treating bacterial disease. A wealth of such compounds exist, since of the c. 3000 antibiotics discovered to date, only a handful of classes have been developed for clinical use. ${ }^{5}$ Of particular interest amongst these unexploited antibiotics are those that have already been documented to possess properties desirable in a potential antibacterial drug to tackle infections caused by the ESKAPE pathogens, for example, broad-spectrum activity and demonstrated antibacterial efficacy in animal models. While a proportion of such compounds will likely have been considered and rejected for development because they possess liabilities that went unreported in the scientific literature, it is also conceivable that many have not been rigorously evaluated as drug candidates. Furthermore, there are several examples of antibiotics that were initially dismissed as drug candidates, but were later revisited and successfully developed for clinical use (for example, daptomycin, fidaxomicin, pleuromutilins). ${ }^{4}$ Thus, we consider a large-scale re-evaluation of known natural product antibiotics to identify antibacterial drug candidates to be a worthwhile endeavor. However, to redress the opacity of past antibacterial drug discovery efforts ${ }^{4}$ and to prevent future duplication of effort, we consider it vital that the scientific record comprehensively captures the details both for compounds that such an analysis reveals to have potential as antibacterial drug candidates, as well as those it does not.

Antibiotic MSD-819 (6-chloro-2-quinoxalinecarboxylic acid 1,4-dioxide; Figure 1a) was isolated from a strain of Streptomyces ambofaciens by Merck Sharp \& Dohme in the late 1960's. 6,7 This compound was shown to exhibit broad-spectrum antibacterial activity in a semiquantitative assay, producing measurable zones of inhibition on agar around filter-paper discs impregnated with MSD-819 ( $1 \mathrm{mg} \mathrm{ml}^{-1}$ ) against organisms that included Enterococcus faecalis, Escherichia coli and some strains of S. aureus. ${ }^{7}$ In vivo activity of MSD-819 was also demonstrated, with intraperitoneal administration of the compound protecting mice infected with a lethal dose of Proteus vulgaris, S. aureus or Salmonella schottmuelleri, at an $\mathrm{ED}_{50}$ of $0.11-1.25 \mathrm{mg}$ per dose. ${ }^{7}$ Furthermore, a carboxamide derivative of MSD-819 was reported to protect mice against lethal infection with P. vulgaris when the compound was administered via the oral route. ${ }^{8}$

To further explore the biological properties of MSD-819, we sought in the first instance to purify the compound from the original producer organism (S. ambofaciens var. MA-2870). ${ }^{7}$ Difficulties encountered in generating sufficient material for evaluation led us instead to obtain the compound by chemical synthesis (Liverpool ChiroChem, Liverpool, UK). The chemical structure and purity $(\geqslant 99 \%)$ of MSD-819 were established by ${ }^{1} \mathrm{H}$ NMR and LC-MS, respectively (Supplementary Figure S1; analysis carried out by Liverpool ChiroChem). The antibacterial activity of MSD-819 was evaluated against E. coli and representative ESKAPE strains. Minimum inhibitory concentration (MIC) determinations, carried out according to the CLSI guidelines for broth susceptibility testing, ${ }^{9}$ revealed MSD-819 to display only weak antibacterial activity against both Gram-positive and Gram-negative bacteria, with MICs of $128 \mathrm{mg} \mathrm{l}^{-1}$ against E. faecium and E. coli (Figure 1b). In accordance with previous findings, MSD-819 was inactive against $P$. aeruginosa and some strains of $S$. aureus. ${ }^{7}$ While restricted intracellular accumulation often explains the poor activity of many antibiotic classes against Gram-negative bacteria, ${ }^{10}$ this phenomenon did not appear to adversely impact the anti-Gram-negative activity of MSD-819; no significant decrease in

Antimicrobial Research Centre and School of Molecular and Cellular Biology, University of Leeds, Leeds, UK

Correspondence: Dr AJ O'Neill, Antimicrobial Research Centre and School of Molecular and Cellular Biology, University of Leeds, Leeds LS2 9JT, UK.

E-mail: a.j.oneill@leeds.ac.uk

Received 6 July 2016; revised 10 October 2016; accepted 23 October 2016; published online 30 November 2016 
<smiles>O=C(O)c1c[n+]([O-])c2cc(Cl)ccc2[n+]1[O-]</smiles>

b

\begin{tabular}{c|c}
\hline Bacterial strain & MIC $(\mathrm{mg} / \mathrm{L})$ \\
\hline Enterococcus faecium MRL 765793 & 128 \\
Staphylococcus aureus SH1000 & $>256$ \\
Staphylococcus aureus ACC A790662 & $>256$ \\
Klebsiella pneumoniae 581346 & 256 \\
Acinetobacter baumannii 581217 & 256 \\
Pseudomonas aeruginosa PA01 & $>256$ \\
Pseudomonas aeruginosa PA14 & $>256$ \\
Enterobacter cloacae 583750 & 256 \\
Escherichia coli IHMA 659048 & 128 \\
Escherichia coli BW25113 & 128 \\
\hline
\end{tabular}

Figure 1 Chemical structure (a) and antibacterial activity (b) of MSD-819.

MIC was observed in E. coli BW25113 ${ }^{11}$ deleted for the AcrAB-TolC transporter, or upon permeabilization with polymyxin B nonapeptide ${ }^{10}$ (data not shown).

The antibacterial properties of MDS-819 were further evaluated against E. coli BW25113. In time-kill experiments, ${ }^{9}$ MSD-819 was bactericidal at $4 \times$ MIC against rapidly growing cells, reducing an exponential phase population of $\sim 5 \times 10^{5} \mathrm{CFU} \mathrm{ml}^{-1}$ to below the limit of detection $\left(1 \mathrm{CFU} \mathrm{ml}{ }^{-1}\right)$ in $24 \mathrm{~h}$ (Figure $2 \mathrm{a}$ ). In common with most antibacterial drugs that exert a cidal action upon rapidly growing bacteria, the compound had negligible effect on the viability of nongrowing cultures (bacteria from a stationary phase [16 h] culture, re-suspended in spent medium to a cell density of $\sim 5 \times 10^{5}$ $\mathrm{CFU} \mathrm{ml}{ }^{-1}$ ) (Figure 2a). To evaluate the potential for MSD-819 to select resistance, concentrated cultures of $E$. coli were spread onto agar containing the antibiotic at $4 \times$ MIC. $^{12}$ While the comparator agent rifampicin selected mutants with reduced susceptibility at a frequency of $\sim 2 \times 10^{-8}$, no mutants resistant to MSD-819 could be recovered (limit of detection: $1.5 \times 10^{-10}$ ). Consequently, we consider MSD-819 to have low mutational resistance potential. ${ }^{13}$

The majority of antibiotics in clinical use exert their antibacterial effects by interfering with the biosynthesis of cellular macromolecules. ${ }^{13}$ We therefore initiated our investigation into the antibacterial mode of action of MSD-819 by evaluating the effect of the compound on the incorporation of radiolabelled precursors into DNA, RNA and protein in E. coli. ${ }^{14}$ At $4 \times$ MIC, MSD-819 achieved $>95 \%$ inhibition of all three macromolecular biosynthesis pathways within $10 \mathrm{~min}$, while the comparator agents ciprofloxacin, rifampicin and tetracycline exhibited the expected preferential inhibition of DNA, RNA and protein synthesis, respectively (Figure 2b). Non-specific inhibition of macromolecular biosynthesis is a characteristic of compounds that exert their antibacterial effects through perturbation of the cytoplasmic membrane. ${ }^{15-17}$ We therefore evaluated the effect of MSD-819 on bacterial membrane integrity, alongside comparator agents, using the Live/Dead BacLight kit (Invitrogen, Paisley, UK). ${ }^{18}$
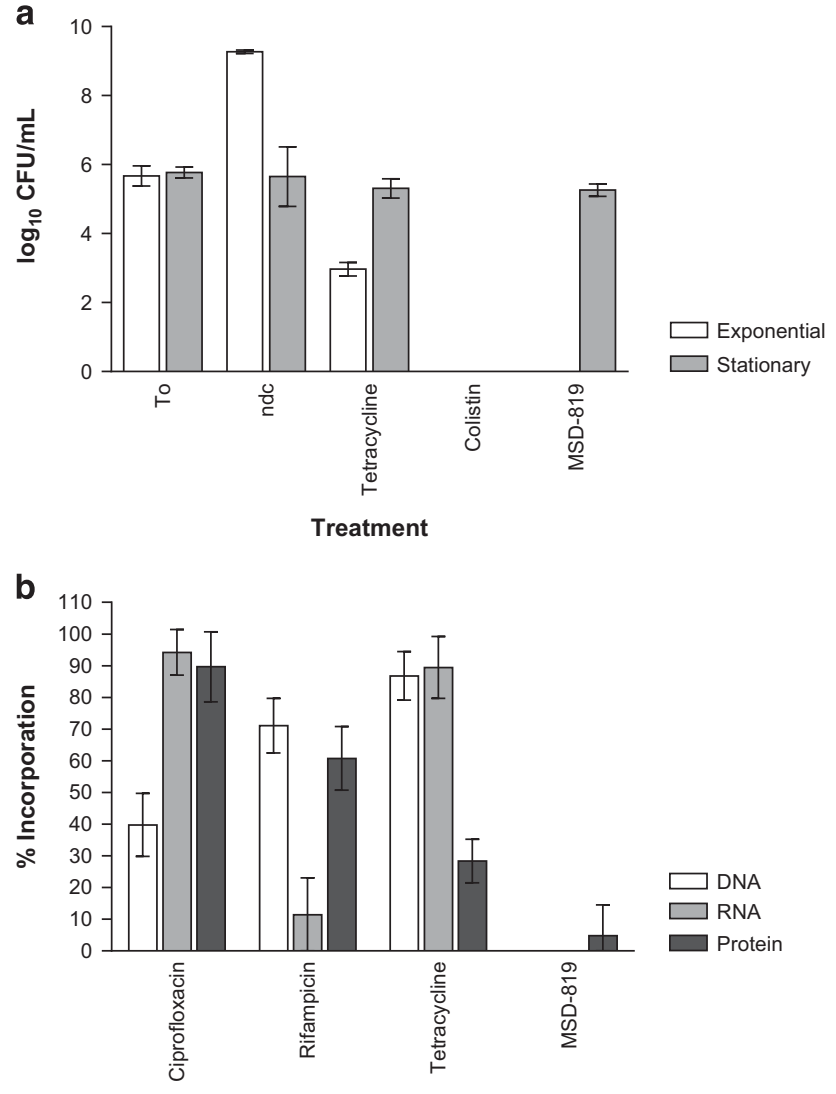

Antimicrobial agent

Figure 2 Effects of MSD-819 and comparator agents at $4 \times$ MIC on viability and macromolecular biosynthesis pathways of E. coli BW25113. (a) Viability of exponential and stationary phase cultures of $E$. coli exposed to compounds for $24 \mathrm{~h}$ (level of detection, $1 \mathrm{CFU} \mathrm{ml}^{-1}$ ). NDC - no drug control. (b) Effect of a 10 min exposure of $E$. coli to antibacterial agents on incorporation of radiolabeled precursors into macromolecules. The datum points represent the means of at least three independent experiments.

Incubation of E. coli with MSD-819 at $4 \times$ MIC caused essentially complete loss of membrane integrity $(99.5 \pm 0.3 \%)$ in $10 \mathrm{~min}$, a similar result to that recorded for the known membrane-active agent, colistin $(94.1 \pm 5.1 \%)$. By contrast, the non-membrane active antibiotic tetracycline caused no significant reduction in membrane integrity $(7.5 \pm 9.2 \%)$.

Compounds that act to perturb bacterial membranes often exert similar effects on mammalian membranes, an undesirable property for an antibacterial drug candidate. To evaluate the specificity of MSD-819 for bacterial membranes, we challenged equine erythrocytes with $4 \times$ MIC of MSD- 819 for $60 \mathrm{~min}$ and monitored membrane integrity by measuring leakage of hemoglobin at an absorbance of $540 \mathrm{~nm} .{ }^{16}$ MSD-819 caused a substantially greater loss of mammalian membrane integrity $(39.9 \pm 2.5 \%)$ than observed for the comparator agents, colistin and tetracycline $(16.6 \pm 4.5 \%$ and $4.0 \pm 4.0 \%$, respectively). We subsequently examined whether the ability of MSD-819 to perturb mammalian membranes was associated with cytotoxic effects on mammalian cells. Cytotoxicity was evaluated in human kidney- 2 cells by measuring ATP levels (using the Adenosine $5^{\prime}$-triphosphate bioluminescent somatic cell assay kit, Sigma-Aldrich) after $6 \mathrm{~h}$ challenge with MDS-819. MSD-819 displayed an $\mathrm{EC}_{50}$ of $<256 \mathrm{mg} \mathrm{l}^{-1}$, indicative of cytotoxic effects at concentrations close to the bacterial MIC, implying that MSD-819 lacks prokaryotic selectivity. 
Most antibacterial drugs approved for systemic use have an MIC below, or close to, $1 \mathrm{mgl}^{-1} .13$ While the antibacterial activity of MSD-819 against the ESKAPE pathogens is weak in comparison, this should not alone rule this class of compound from further consideration as a candidate for antibacterial chemotherapy, particularly since chemical derivatization has been shown to boost its antibacterial potency by $>20$-fold. ${ }^{8}$ However, the finding that the membrane-perturbing mechanism by which MSD-819 exerts its antibacterial effect also negatively impacts mammalian membranes, and that the compound therefore lacks selectivity of action against bacteria, indicates that MSD-819 does not represent a promising candidate for medicinal chemistry to improve its antibacterial potency. Future studies will seek to evaluate other previously unexploited antibiotic classes to assess their potential as antibacterial drug candidates.

\section{CONFLICT OF INTEREST}

The authors declare no conflict of interest.

\section{ACKNOWLEDGEMENTS}

We thank R Gonciarz (University of Leeds) for advice and for providing the figure of MSD-819. This work was supported by grant MR/L000369/1 from the Medical Research Council (UK).

1 O'Neill, J. Antimicrobial resistance: tackling a crisis for the health and wealth of nations. Rev. Antimicrob. Resist. (2014).

2 Talbot, G. H. et al. Bad bugs need drugs: an update on the development pipeline from the Antimicrobial Availability Task Force of the Infectious Diseases Society of America. Clin. Infect. Dis. 42, 657-668 (2006).

3 CDC. Antibiotic resistance threats in The United States. Available at http://www.cdc. gov/drugresistance/pdf/ar-threats-2013-508.pdf (2013).

4 Silver, L. L. Challenges of antibacterial discovery. Clin. Microbiol. Rev. 24, 71-109 (2011).

5 Lewis, K. Platforms for antibiotic discovery. Nat. Rev. Drug Disc. 12, 371-387 (2013)

6 Miller, T. W., Walker, R. W., Trenner, N. R., Arison, B. A. \& Wolf, F. J. Antibiotic MSD819. II. Isolation and chemical characterization as 6-chloro-2-quinoxalinecarboxylic acid 1,4-dioxide. Antimicrob. Agents Chemother. (Bethesda, MD, USA) 8, 255-259 (1968).
7 Stapley, E. O. et al. Antibiotic MSD-819. I. Microbial production and biological characteristics. Antimicrob. Agents Chemother. (Bethesda, MD, USA) 8 249-254 (1968).

8 Miller, T. W., Miller, A. K., Wolf, F. J., Stapley, E. O. \& Hendlin, D. Antibiotic MSD-819. Preparation and properties of biologically active derivatives. J. Antibiot. 22, 293-296 (1969).

9 CLSI. Methods for Dilution Antimicrobial Susceptibility Tests for Bacteria That Grow Aerobically. 9th edn, Vol. 32. Approved standard M07-A9, http://clsi.org/ (The Clinical and Laboratory Standards Institute, 2012).

10 Randall, C. P., Mariner, K. R., Chopra, I. \& O'Neill, A. J. The target of daptomycin is absent from Escherichia coli and other Gram-negative pathogens. Antimicrob. Agents Chemother. 57, 637-639 (2013).

11 Baba, T. et al. Construction of Escherichia coli K-12 in-frame, single-gene knockout mutants: the Keio collection. Mol. Sys. Biol. 2, 0008 (2006).

12 Ryder, V. J., Chopra, I. \& O'Neill, A. J. Increased mutability of Staphylococci in biofilms as a consequence of oxidative stress. PLOS ONE 7, e47695 (2012).

13 O'Neill, A. J. \& Chopra, I. Preclinical evaluation of novel antibacterial agents by microbiological and molecular techniques. Expert Opin. Invest. Drug. 13, 1045-1063 (2004).

14 Wilson, M., Oliva, B. Cassels, R., Ohanlon, P. J. \& Chopra, I. SB-205952, a novel semisynthetic monic acid analog with at least two modes of action. Antimicrob. Agents Chemother. 39, 1925-1933 (1995).

15 Oliva, B., O'Neill, A. J., Miller, K., Stubbings, W. \& Chopra, I. Antistaphylococcal activity and mode of action of clofazimine. J. Antimicrob. Chemother. 53, 435-440 (2004).

16 Ooi, N. et al. Antibacterial activity and mode of action of tert-butylhydroquinone (TBHQ) and its oxidation product, tert-butylbenzoquinone (TBBQ). J. Antimicrob. Chemother 68, 1297-1304 (2013).

17 Randall, C. P., Oyama, L. B., Bostock, J. M., Chopra, I. \& O'Neill, A. J. The silver cation (Ag+): antistaphylococcal activity, mode of action and resistance studies. J. Antimicrob. Chemother. 68, 131-138 (2013).

18 O'Neill, A. J., Miller, K., Oliva, B. \& Chopra, I. Comparison of assays for detection of agents causing membrane damage in Staphylococcus aureus. J. Antimicrob. Chemother. 54, 1127-1129 (2004).

This work is licensed under a Creative Commons Attribution 4.0 International License. The images or other third party material in this article are included in the article's Creative Commons license, unless indicated otherwise in the credit line; if the material is not included under the Creative Commons license, users will need to obtain permission from the license holder to reproduce the material. To view a copy of this license, visit http:// creativecommons.org/licenses/by/4.0/

(C) The Author(s) 2017

Supplementary Information accompanies the paper on The Journal of Antibiotics website (http://www.nature.com/ja) 